\title{
Systematic study of spontaneous emission in a two-dimensional arbitrary inhomogeneous environment
}

\author{
Peng-fei Qiao, Wei E. I. Sha, and Wallace C. H. Choy \\ Department of Electrical and Electronic Engineering, The University of Hong Kong, Pokfulam Road, Hong Kong \\ Weng Cho Chew ${ }^{\dagger}$ \\ Department of Electrical and Electronic Engineering, The University of Hong Kong, Pokfulam Road, Hong Kong and Department \\ of Electrical and Computer Engineering, University of Illinois at Urbana-Champaign, Urban, Illinois 61820, USA
}

(Received 10 July 2010; published 20 April 2011)

\begin{abstract}
The spontaneous emission (SE) of the excited atoms in a two-dimensional (2D) arbitrary inhomogeneous environment has been systematically studied. The local density of states, which determines the radiation dynamics of a point source (for 3D) or a line source (for 2D), in particular, the SE rate, is represented by the electric dyadic Green's function. The numerical solution of the electric Green's tensor has been accurately obtained with the finite-difference frequency-domain method with the proper approximations of the monopole and dipole sources. The SE of atoms in photonic crystal and plasmonic metal plates has been comprehensively and comparatively investigated. For both the photonic crystal and plasmonic plates systems, the SEs depend on their respective dispersion relations and could be modified by the finite-structure or finite-size effects. This work is important for SE engineering and the optimized design of optoelectronic devices.
\end{abstract}

DOI: 10.1103/PhysRevA.83.043824

PACS number(s): 42.50.Ct, 31.15.xf, 42.50.Nn, 42.50.Pq

\section{INTRODUCTION}

Control of spontaneously emitted light lies at the heart of quantum optics. It is essential for diverse applications such as lasers, light-emitting diodes (LEDs), solar cells, and quantum information [1,2]. It is well known that the radiation dynamics of an atom strongly depends on its environment, which was first discovered by Purcell [3], and the spontaneous emission (SE) can be enhanced if the emitting atom is coupled to a cavity resonator. According to the quantum electrodynamics theory, the SE of an atom can be a weak-coupling radiation process due to the vacuum fluctuations of the electromagnetic field. A suitable modification of an inhomogeneous environment is required so that the vacuum fluctuations controlling the SE can be manipulated. Inhibiting unwanted SE and boosting desired ones will promote novel optoelectronic designs tailored to industrial standards. The local density of states (LDOS) counts the number of electromagnetic modes where photons can be emitted at the specific location of the emitter and can be interpreted as the density of vacuum fluctuations. The inhibition or enhancement of SE reduces to how the LDOS of photons is controlled.

Bragg scattering in the photonic crystal (PC) can yield a photonic band gap that prohibits light propagation over a range of frequencies and forms the atom-photon "bound states" $[4,5]$. The LDOS of photons within the band gap is inhibited, and therefore, the SE of an atom is forbidden. Placing an active medium within the PC cavity can prohibit undesired SE and allow emission only into the lasing mode, thus dramatically improving the efficiency of the laser [2]. Another example is that the external quantum efficiency of an LED can be significantly improved by introducing a two-dimensional photonic crystal structure [6]. Moveover, a

\footnotetext{
*wsha@eee.hku.hk

†wcchew@hku.hk
}

sharp band edge of a PC produces new physical phenomena, such as the coherent control of SE [7] and the non-Markovian quantum fluctuations and superradiance [8]. However, the challenge is on how to calculate the LDOS of photons in a variety of PC structures. The most commonly adopted method is the plane-wave expansion algorithm [9]. This algorithm expands the electromagnetic field as the summation of plane waves and recasts Maxwell's equations into an eigenvalue problem. Then the LDOS can be obtained by the Brillouin zone integrals. Due to the slow convergence, the algorithm always needs a large number of summation terms to guarantee the desired accuracy. Moreover, the algorithm implicitly imposes the periodic boundary conditions for the infinite PC structure. Hence, the plane-wave expansion algorithm suffers from the finite-structure problem or quasiperiodic problem.

The LDOS can also be modified when semiconductor emitters are coupled to the surface plasmon polariton (SPP) of metallic films $[10,11]$. An electron-hole pair emits a photon coupled to a surface plasmon (SP) mode instead of to the free space. The SPP-enhanced SE has been demonstrated in quantum well lasers [12,13], resulting in small effective mode volume. With the presence of strong optical confinement, improved photoluminescence and a low threshold current have been reported. However, most theoretical works employed the approximated methods to model the problem, and convincing results are hard to find in the literature. Furthermore, due to the high contrast and dispersive properties of plasmonic metal materials, the plane-wave expansion algorithm becomes less attractive and effective.

The electric dyadic Green's function (DGF) plays a central role in the theoretical investigation of SE in an arbitrary inhomogeneous and complex environment. The LDOS can thus be expressed in an elegant way. The numerical solution of the electric Green's tensor needs an efficient and rigorous full-wave solver for Maxwell's equations. Although the finite-difference time-domain (FDTD) method $[14,15]$ is a 
candidate to achieve this goal, it has intrinsic disadvantages for modeling the nanopatterned metal structures. First, the complex dielectric constants of metal ( $\mathrm{Au}, \mathrm{Ag}$, etc) have to be described by a large number of summation terms in the Lorentz-Drude model [16], and thus, the recursive convolution implementation is very expensive. Second, the FDTD method will suffer from an instability problem if flexible spatial discretization forms are applied [17].

In this paper, the finite-difference frequency-domain (FDFD) method [18,19] is adopted to obtain the electric DGF in two-dimensional (2D) arbitrary inhomogeneous environments. In particular, we explore the proper discretization strategy to express the 2D line sources involving the monopole source and the dipole source. The spontaneous emission rates (SERs) in the finite photonic crystal structure and finite-size plasmonic metal plates have been comprehensively and comparatively investigated. According to our survey, the SEs of both systems strongly depend on their respective dispersion relations. This conclusion provides a universal concept that the SE can be tailored by dispersion management. Additionally, the dispersion relations could be modified by the finite-structure or finite-size effects.

\section{THEORETICAL PRINCIPLES}

\section{A. Spontaneous emission rate}

Due to the vacuum fluctuations of the electromagnetic field, an atom or molecule embedded in an inhomogeneous medium spontaneously emits photons. Let us consider a single atom located at $\mathbf{r}_{0}$ and represented by a two-level system with the transition dipole moment $\mathbf{p}$ and transition frequency $\omega_{0}$. Considering weak excitation, according to Fermi's golden rule [20], the SER of the atom can be expressed as

$$
\gamma\left(\mathbf{r}_{0}, \omega_{0}\right)=\frac{\pi \omega_{0}}{\epsilon_{0} \hbar} \sum_{k}\left[\mathbf{p} \cdot\left(\mathbf{u}_{\mathbf{k}} \mathbf{u}_{\mathbf{k}}^{*}\right) \cdot \mathbf{p}\right] \delta\left(\omega_{\mathrm{k}}-\omega_{0}\right),
$$

where $\mathbf{u}_{\mathbf{k}}\left(\mathbf{r}_{0}, \omega_{0}\right)$ are the orthonormal eigenmodes of photons in an inhomogeneous medium. Using the eigenfunction expansion technique, the electric DGF can be expressed as the summation of orthonormal eigenmodes [21,22].

$$
\operatorname{Im}\left[\overline{\mathbf{G}}\left(\mathbf{r}, \mathbf{r}^{\prime}, \omega_{0}\right)\right]=\frac{\pi c^{2}}{2 \omega_{0}} \sum_{k} \mathbf{u}_{\mathbf{k}}\left(\mathbf{r}, \omega_{k}\right) \mathbf{u}_{\mathbf{k}}^{*}\left(\mathbf{r}^{\prime}, \omega_{k}\right) \delta\left(\omega_{k}-\omega_{0}\right)
$$

This equation can also be derived with the fluctuationdissipation theorem [23], which writes the imaginary part of the electric Green's tensor in terms of the power spectrum of the electric field in vacuum.

Substituting Eq. (2) into Eq. (1), we get

$$
\gamma\left(\mathbf{r}_{0}, \omega_{0}\right)=\frac{2 \omega_{0}^{2}}{\epsilon_{0} \hbar c^{2}}\left\langle\mathbf{p} \cdot \operatorname{Im}\left[\overline{\mathbf{G}}\left(\mathbf{r}_{0}, \mathbf{r}_{0}, \omega_{0}\right)\right] \cdot \mathbf{p}\right\rangle .
$$

If the transitions of the quantum system have no fixed dipole axis and the medium is isotropic, the SER needs to be averaged over various orientations leading to

$$
\gamma\left(\mathbf{r}_{0}, \omega_{0}\right)=\frac{2 \omega_{0}^{2}|\mathbf{p}|^{2}}{3 \epsilon_{0} \hbar c^{2}} \operatorname{Im}\left\{\operatorname{Tr}\left[\overline{\mathbf{G}}\left(\mathbf{r}_{0}, \mathbf{r}_{0}, \omega_{0}\right)\right]\right\}
$$

This suggests that the local coupling of the atomic dipole moment to photons in this mode determines the SER of the excited atom. In other words, the averaged SER is related to the LDOS [20] defined by

$$
\begin{aligned}
\rho\left(\mathbf{r}_{0}, \omega_{0}\right) & =\sum_{\mathbf{k}}\left|\mathbf{u}_{\mathbf{k}}\right|^{2} \delta\left(\omega_{\mathbf{k}}-\omega_{0}\right) \\
& =\frac{2 \omega_{0}}{\pi c^{2}} \operatorname{Im}\left\{\operatorname{Tr}\left[\overline{\mathbf{G}}\left(\mathbf{r}_{0}, \mathbf{r}_{0}, \omega_{0}\right)\right]\right\} .
\end{aligned}
$$

To evaluate the SE enhancement, we can define the normalized SER as

$$
\frac{\gamma}{\gamma_{0}}=\frac{\rho\left(\mathbf{r}_{0}, \omega_{0}\right)}{\rho_{0}\left(\mathbf{r}_{0}, \omega_{0}\right)}=\frac{\operatorname{Im}\left\{\operatorname{Tr}\left[\overline{\mathbf{G}}\left(\mathbf{r}_{0}, \mathbf{r}_{0}, \omega_{0}\right)\right]\right\}}{\operatorname{Im}\left\{\operatorname{Tr}\left[\overline{\mathbf{G}}_{0}\left(\mathbf{r}_{0}, \mathbf{r}_{0}, \omega_{0}\right)\right]\right\}}
$$

where $\overline{\mathbf{G}}_{0}$ indicates the DGF in free space. In fact, the normalized SER is the Purcell factor in quantum electrodynamics.

\section{B. Electric dyadic Green's function in 2D free space}

The electric DGF in free space can be represented as the summation of three vectorial Green's functions,

$$
\overline{\mathbf{G}}\left(\mathbf{r}, \mathbf{r}^{\prime}\right)=\mathbf{G}_{x}\left(\mathbf{r}, \mathbf{r}^{\prime}\right) \hat{a}_{x}+\mathbf{G}_{y}\left(\mathbf{r}, \mathbf{r}^{\prime}\right) \hat{a}_{y}+\mathbf{G}_{z}\left(\mathbf{r}, \mathbf{r}^{\prime}\right) \hat{a}_{z},
$$

which satisfy the vector wave equations,

$$
\begin{gathered}
\nabla \times \nabla \times \mathbf{G}_{m}\left(\mathbf{r}, \mathbf{r}^{\prime}\right)-k_{0}^{2} \mathbf{G}_{m}\left(\mathbf{r}, \mathbf{r}^{\prime}\right)=\hat{a}_{m} \delta\left(\mathbf{r}-\mathbf{r}^{\prime}\right), \\
m=x, y, z,
\end{gathered}
$$

where $\hat{a}_{m} \delta\left(\mathbf{r}-\mathbf{r}^{\prime}\right)$ is the Dirac's delta source at $\mathbf{r}=\mathbf{r}^{\prime}$ with the polarization along the $x, y$, and $z$ directions, respectively. The wave number in free space is denoted as $k_{0}$. For the $z-$ invariant $2 \mathrm{D}$ problem, the source considered here is actually a line source, and the 2D electric DGF can be simplified as

$$
\overline{\mathbf{G}}\left(\mathbf{r}, \mathbf{r}^{\prime}\right)=\left(\begin{array}{ccc}
G_{x x} & G_{y x} & 0 \\
G_{x y} & G_{y y} & 0 \\
0 & 0 & G_{z z}
\end{array}\right)
$$

and the TM ( $E_{z}$ field) and TE $\left(H_{z}\right.$ field) modes decouple with each other.

For pointlike emitters, such as a quantum dot, the SE can be analyzed by computing the 3D electric DGF [24]. However, for optoelectronic devices, including LEDs and lasers, the gain media are always embedded into the $z$-invariant emitting layers [25]. Hence, the gain media can be viewed as linelike emitters. Mathematically, the 2D DGF can be connected with the 3D DGF by the following identity:

$$
\frac{i}{4} H_{0}^{(1)}\left(k_{0}\left|\mathbf{t}-\mathbf{t}^{\prime}\right|\right)=\int_{-\infty}^{\infty} \frac{\exp \left(i k_{0}\left|\mathbf{r}-\mathbf{r}^{\prime}\right|\right)}{4 \pi\left|\mathbf{r}-\mathbf{r}^{\prime}\right|} d z^{\prime},
$$

where $\mathbf{r}=\mathbf{t}+z \hat{a}_{z}$ and $\mathbf{r}^{\prime}=\mathbf{t}^{\prime}+z^{\prime} \hat{a}_{z}$. From this identity, we may understand that the SE of a line emitter is the ensemble accumulation of that of point emitters. In recent works, the outgoing power of 1D multilayered LED structure calculated by planar source (not point source) shows good agreement with 
the experimental result [26]. In addition, the Casimir force between $z$-invariant nanostructures by vacuum fluctuations of the electromagnetic field is also characterized by the $2 \mathrm{D}$ DGFs [27,28]. In this paper, we will study the SE in 2D finite structures, and hence, line source is employed. For this point, more theoretical and experimental studies should be done in the future.

The component $G_{z z}$ in Eq. (9) can be found by solving the TM scalar wave equation of the $E_{z}$ field in free space:

$$
\nabla^{2} E_{z}+k_{0}^{2} E_{z}=-i \omega \mu_{0} J_{z}=-\delta,
$$

where $\delta=\delta\left(x-x^{\prime}\right) \delta\left(y-y^{\prime}\right)$ is the $z$-polarized monopole source and $E_{z}=E_{z}(x, y)$.

The components $G_{x x}$ and $G_{y y}$ can be found by solving the TE scalar wave equation of $H_{z}$ field in free space:

$$
\begin{gathered}
\nabla^{2} H_{z}+k_{0}^{2} H_{z}=\frac{\partial J_{x}}{\partial y}=\frac{1}{i \omega \mu_{0}} \frac{\partial \delta}{\partial y}, \\
\nabla^{2} H_{z}+k_{0}^{2} H_{z}=\frac{-\partial J_{y}}{\partial x}=\frac{-1}{i \omega \mu_{0}} \frac{\partial \delta}{\partial x},
\end{gathered}
$$

where $\partial \delta / \partial y$ and $\partial \delta / \partial x$ are the $x$-polarized and $y$-polarized dipole sources. After getting the $H_{z}$ field component, $G_{x x}$ is obtained from the $E_{x}$ field generated by the dipole source, i.e.,

$$
G_{x x}=E_{x}=\frac{1}{k_{0}^{2}}\left(i \omega \mu_{0} \frac{\partial H_{z}}{\partial y}-\delta\right) .
$$

Similarly, $G_{y y}$ can be obtained from

$$
G_{y y}=E_{y}=\frac{1}{k_{0}^{2}}\left(-i \omega \mu_{0} \frac{\partial H_{z}}{\partial x}-\delta\right) .
$$

The analytical solution of the electric DGF in 2D free space is important for validating our theoretical model. We can see that the imaginary part of the electric DGF at the origin is regular, which agrees with the fact that the SER is finite.

$$
\begin{aligned}
\operatorname{Im}\left[G_{z z}\left(\mathbf{t}_{\mathbf{0}}, \mathbf{t}_{\mathbf{0}}\right)\right]= & \lim _{\mathbf{t} \rightarrow \mathbf{t}^{\prime}} \operatorname{Im}\left[\frac{i}{4} H_{0}^{(1)}\left(k_{0}\left|\mathbf{t}-\mathbf{t}^{\prime}\right|\right)\right] \\
= & \frac{1}{4} \lim _{\mathbf{t} \rightarrow \mathbf{t}^{\prime}} J_{0}\left(k_{0}\left|\mathbf{t}-\mathbf{t}^{\prime}\right|\right) \\
= & 0.25, \\
\operatorname{Im}\left[G_{x x}\left(\mathbf{t}_{\mathbf{0}}, \mathbf{t}_{\mathbf{0}}\right)\right]= & \lim _{\mathbf{t} \rightarrow \mathbf{t}^{\prime}} \frac{-1}{4 k_{0}^{2}} \frac{\partial^{2}}{\partial y^{2}} J_{0}\left(k_{0}\left|\mathbf{t}-\mathbf{t}^{\prime}\right|\right) \\
= & \lim _{\mathbf{t} \rightarrow \mathbf{t}^{\prime}}\left[\frac{1}{4 k_{0}\left|\mathbf{t}-\mathbf{t}^{\prime}\right|} J_{1}\left(k_{0}\left|\mathbf{t}-\mathbf{t}^{\prime}\right|\right)\right. \\
& \left.-\frac{\left(y-y^{\prime}\right)^{2}}{4\left|\mathbf{t}-\mathbf{t}^{\prime}\right|^{2}} J_{2}\left(k_{0}\left|\mathbf{t}-\mathbf{t}^{\prime}\right|\right)\right] \\
= & 0.125, \\
\operatorname{Im}\left[G_{y y}\left(\mathbf{t}_{\mathbf{0}}, \mathbf{t}_{\mathbf{0}}\right)\right]= & \lim _{\mathbf{t} \rightarrow \mathbf{t}^{\prime}} \frac{-1}{4 k_{0}^{2}} \frac{\partial^{2}}{\partial x^{2}} J_{0}\left(k_{0}\left|\mathbf{t}-\mathbf{t}^{\prime}\right|\right) \\
= & \lim _{\mathbf{t} \rightarrow \mathbf{t}^{\prime}}\left[\frac{1}{4 k_{0}\left|\mathbf{t}-\mathbf{t}^{\prime}\right|} J_{1}\left(k_{0}\left|\mathbf{t}-\mathbf{t}^{\prime}\right|\right)\right. \\
& \left.-\frac{\left(x-x^{\prime}\right)^{2}}{4\left|\mathbf{t}-\mathbf{t}^{\prime}\right|^{2}} J_{2}\left(k_{0}\left|\mathbf{t}-\mathbf{t}^{\prime}\right|\right)\right] \\
= & 0.125 .
\end{aligned}
$$

\section{Finite-difference frequency-domain method}

In order to characterize the SE in an arbitrary inhomogeneous environment, a rigorous, accurate, and efficient solver for Maxwell's equations is essential. Compared with other algorithms, the FDFD method is very easy to implement and has a good ability to handle frequency-dependent material properties. Moreover, it can treat various inhomogeneous boundary conditions conveniently. Finally, the matrix produced by the FDFD method is sparse and can be solved with the multigrid fast algorithm [29] with a memory cost of $O(N)$ and a complexity of $O(N)$ for some problems.

With the Yee lattice [30], the 2D FDFD method is utilized to obtain the electric DGF. We employ the perfectly matched layer (PML) [31,32] as the absorbing boundary condition to truncate the computational region. The discretization forms of $\mathrm{TM} / \mathrm{TE}$ wave equations with averaged dielectric constants are also derived.

The numerical treatment of the monopole and dipole sources plays a key role in calculating the electric DGF. For the 2D monopole source, we have

$$
\left.\delta\left(x-m^{\prime} \Delta_{x}, y-n^{\prime} \Delta_{y}\right)\right|_{x=m^{\prime} \Delta_{x}, y=n^{\prime} \Delta_{y}} \approx \frac{1}{\Delta_{x} \Delta_{y}},
$$

where $\Delta_{x}$ and $\Delta_{y}$ are the spatial steps along the $x$ and $y$ directions, respectively, and $\left(m^{\prime}, n^{\prime}\right)$ is the grid index of the source point of the $E_{z}$ component. If we regard the monopole source as a rectangular pulse function and apply the finite-difference technique again, the dipole source can be approximated as

$$
\begin{array}{r}
\frac{\partial \delta\left(x-m^{\prime} \Delta_{x}, y-n^{\prime} \Delta_{y}\right)}{\partial y} \\
\approx-\frac{\delta\left(x-m^{\prime} \Delta_{x}, y-\left(n^{\prime}+0.5\right) \Delta_{y}\right)}{\Delta_{y}} \\
\quad+\frac{\delta\left(x-m^{\prime} \Delta_{x}, y-\left(n^{\prime}-0.5\right) \Delta_{y}\right)}{\Delta_{y}}
\end{array}
$$

This can be simplified to $-1 /\left(\Delta_{x} \Delta_{y}^{2}\right)$ and $1 /\left(\Delta_{x} \Delta_{y}^{2}\right)$ at the source points $\left(m^{\prime}, n^{\prime}+0.5\right)$ and $\left(m^{\prime}, n^{\prime}-0.5\right)$ of the $H_{z}$ components, respectively.

The wave equations for the TM and TE modes in an inhomogeneous environment are respectively formulated as

$$
\begin{aligned}
& \frac{1}{\epsilon_{r}} \frac{\partial}{\partial x}\left(\frac{1}{\mu_{r}} \frac{\partial E_{z}}{\partial x}\right)+\frac{1}{\epsilon_{r}} \frac{\partial}{\partial y}\left(\frac{1}{\mu_{r}} \frac{\partial E_{z}}{\partial y}\right)+k_{0}^{2} E_{z}=0, \\
& \frac{1}{\mu_{r}} \frac{\partial}{\partial x}\left(\frac{1}{\epsilon_{r}} \frac{\partial H_{z}}{\partial x}\right)+\frac{1}{\mu_{r}} \frac{\partial}{\partial y}\left(\frac{1}{\epsilon_{r}} \frac{\partial H_{z}}{\partial y}\right)+k_{0}^{2} H_{z}=0,
\end{aligned}
$$

where $\epsilon_{r}$ and $\mu_{r}$ are the relative permittivities and permeabilities. In this paper, we assume that $\mu_{r}=1, \epsilon_{r}=n_{c}^{2}$, and $n_{c}$ is the complex dielectric constant of the optical material.

As shown in Fig. 1, a five-point stencil is used for the FDFD method. The discretized forms for the TM and TE wave 


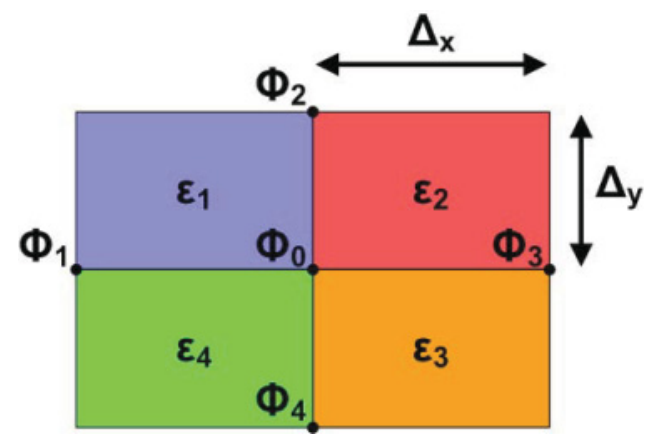

FIG. 1. (Color online) The five-point stencil for the FDFD method. $\Delta_{x}$ and $\Delta_{y}$ are, respectively, the spatial steps along the $x$ and $y$ directions. $\Phi=E_{z}$ for a TM wave, and $\Phi=H_{z}$ for a TE wave. $\epsilon=n_{c}^{2}$ is the relative permittivity in the discretized region, and $n_{c}$ is the complex dielectric constant of the optical material.

equations are respectively of the form

$$
\begin{gathered}
2\left(\frac{1}{\Delta_{x}^{2}}+\frac{1}{\Delta_{y}^{2}}\right) \frac{\Phi_{0}}{\bar{\epsilon}}-k_{0}^{2} \Phi_{0}-\frac{\Phi_{1}+\Phi_{3}}{\bar{\epsilon} \Delta_{x}^{2}}-\frac{\Phi_{2}+\Phi_{4}}{\bar{\epsilon} \Delta_{y}^{2}}=0, \\
\Phi=E_{z},
\end{gathered}
$$

$$
\begin{gathered}
2\left(\frac{1}{\Delta_{x}^{2}}+\frac{1}{\Delta_{y}^{2}}\right) \frac{\Phi_{0}}{\bar{\epsilon}}-k_{0}^{2} \Phi_{0}-\frac{\epsilon_{1}^{-1}+\epsilon_{4}^{-1}}{2 \Delta_{x}^{2}} \Phi_{1}-\frac{\epsilon_{2}^{-1}+\epsilon_{3}^{-1}}{2 \Delta_{x}^{2}} \Phi_{3} \\
-\frac{\epsilon_{1}^{-1}+\epsilon_{2}^{-1}}{2 \Delta_{y}^{2}} \Phi_{2}-\frac{\epsilon_{3}^{-1}+\epsilon_{4}^{-1}}{2 \Delta_{y}^{2}} \Phi_{4}=0 \\
\Phi=H_{z}
\end{gathered}
$$$$
\bar{\epsilon}= \begin{cases}\frac{\epsilon_{1}+\epsilon_{2}+\epsilon_{3}+\epsilon_{4}}{4}, & \Phi=E_{z}, \\ 4\left(\epsilon_{1}^{-1}+\epsilon_{2}^{-1}+\epsilon_{3}^{-1}+\epsilon_{4}^{-1}\right)^{-1}, & \Phi=H_{z} .\end{cases}
$$

The PML can absorb the outgoing waves without spurious reflections and is very suitable for the unbounded optical problem. The wave equation with the complex-coordinate- stretched PML [32] is given by

$$
\frac{1}{s_{r}(x)} \frac{\partial}{\partial x}\left(\frac{1}{s_{r}(x)} \frac{\partial \Phi}{\partial x}\right)+\frac{1}{s_{r}(y)} \frac{\partial}{\partial y}\left(\frac{1}{s_{r}(y)} \frac{\partial \Phi}{\partial y}\right)+k_{0}^{2} \Phi=0,
$$

where $s_{r}=1+i \sigma / \omega \epsilon$ and the conductivities $\sigma(x)$ and $\sigma(y)$ are nonzeros only within PML layers normal to the $x$ axis and $y$ axis, respectively. The optimized conductivities [33] in the PML layers are chosen as

$$
\begin{aligned}
& \sigma_{m}=\frac{0.02}{\Delta}\left(\frac{2 m-1}{16}\right)^{3.7}, \quad m=1, \ldots, 8 \\
& \sigma_{m+0.5}=\frac{0.02}{\Delta}\left(\frac{2 m}{16}\right)^{3.7}, \quad m=0, \ldots, 8
\end{aligned}
$$

where $\Delta=\Delta_{x}$ or $\Delta=\Delta_{y}$ for the PML layers normal to the $x$ axis or $y$ axis and $m$ is the grid index of the eight-layer PML.

\section{Benchmark}

To validate our theoretical model, we calculate the electric DGF in 2D free space by using the FDFD method. Making use of the example, we could verify that the FDFD method can accurately calculate the DGF, especially when the source point and the observation point overlap with each other and the singularity arises $\left(\mathbf{r}=\mathbf{r}^{\prime}\right)$. For a given frequency, the DGFs along a fixed direction are calculated with the spatial step of $1 / 20$ wavelength. The FDFD results are compared to the analytical solutions derived in Eqs. (16)-(18). As shown in Figs. 2(a) and 2(b), the numerical results agree with the analytical solutions very well. Figure 2(c) shows the relative errors of the DGFs as a function of points per wavelength. The second-order convergence of the FDFD method still can be guaranteed at the singularity points of the DGFs.

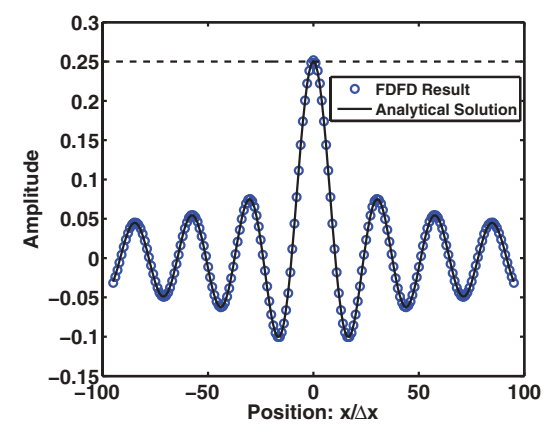

(a)

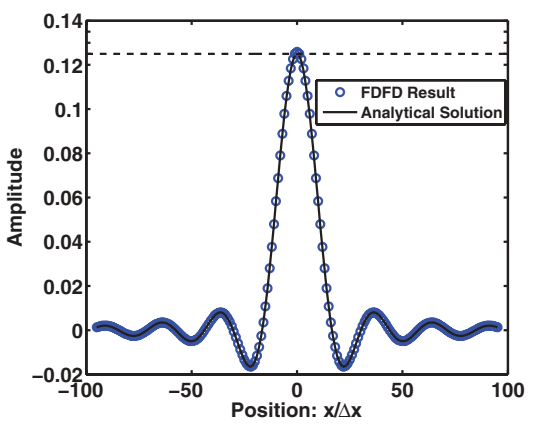

(b)

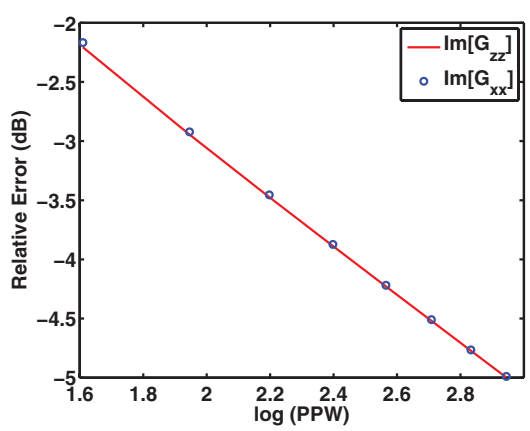

(c)

FIG. 2. (Color online) The imaginary parts of the electric DGFs in 2D free space along the $y=0$ direction. The line source is located at $(x=0, y=0)$. (a) The imaginary part of the $E_{z}$ field generated by a $z$-polarized monopole source. The analytical solution is $0.25 J_{0}(k|x|)$ and $\operatorname{Im}\left[G_{z z}\left(\mathbf{t}_{\mathbf{0}}, \mathbf{t}_{\mathbf{0}}\right)\right]=0.25$. (b) The imaginary part of the $E_{x}$ field generated by an $x$-polarized dipole source. The analytical solution is $J_{1}(k|x|) / 4 k_{0}|x|$ and $\operatorname{Im}\left[G_{x x}\left(\mathbf{t}_{\mathbf{0}}, \mathbf{t}_{\mathbf{0}}\right)\right]=0.125$. (c) The relative errors of the DGFs at the singularity points as a function of points per wavelength (PPW) plotted on the log-log scale. 
(a)

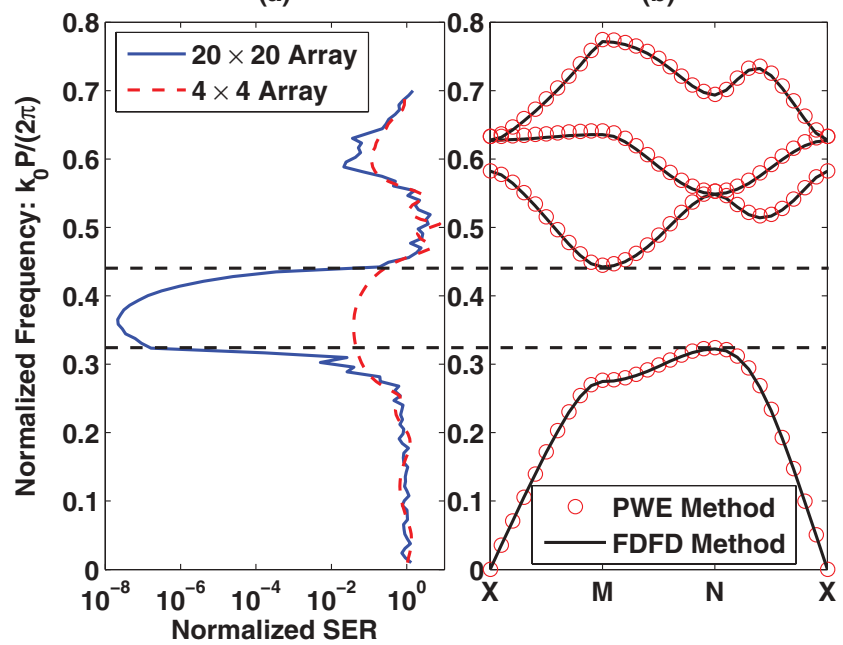

FIG. 3. (Color online) (a) The normalized SER of the atoms, which are located at the air and surrounded by the $4 \times 4$ or $20 \times 20$ dielectric rods. (b) The dispersion curve of the infinite PC structure with dielectric rods for the TM mode. The SER is calculated by $\operatorname{Im}\left[G_{z z}\left(\mathbf{t}_{\mathbf{0}}, \mathbf{t}_{\mathbf{0}}\right)\right]$.

\section{SIMULATION RESULTS}

The SE in different inhomogeneous environments is studied by calculating the electric DGF.

(1) The SE in the square-lattice PCs with the $4 \times 4$ and $20 \times 20$ rods are investigated. In contrast to the plane-wave expansion algorithm, which uses the summation of orthonormal eigenmodes via Eq. (1), the FDFD method characterizes the SE by computing the electric DGF for the finite lattice directly. The dispersion relations of the infinite 2D PC structures are obtained by solving the eigenfrequencies versus the wave vectors on the boundaries of the irreducible Brillouin zone, as shown in Figs. 3(b) and 4(b). For the dispersion curves, $X$ corresponds to $\left(k_{x}=0, k_{y}=0\right), M$ corresponds to $\left(k_{x}=\right.$ $\left.\pi / P, k_{y}=0\right)$, and $N$ corresponds to $\left(k_{x}=\pi / P, k_{y}=\pi / P\right)$. We also calculate the dispersion relations with the FDFD method, imposing the periodic boundary conditions on the unit cell. The dispersion curves calculated with the FDFD method agree with those obtained with the plane-wave expansion algorithm, which demonstrates the powerful capability of the FDFD method in handling inhomogeneous boundary conditions.

The periodicity of the PC structures is denoted by $P$. For the TM case, the radius of the dielectric rod is $0.2 P$, and the dielectric contrast is 8.9 . For the TE case, the radius of the air rod is $0.46 P$, and the dielectric contrast is 12 . In Fig. 3, the dispersion curve of the periodic dielectric rods for the TM mode is compared to the normalized $\operatorname{Im}\left[G_{z z}\left(\mathbf{t}_{\mathbf{0}}, \mathbf{t}_{\mathbf{0}}\right)\right]$, which is also the normalized SER of the longitudinal $(z-)$ polarized atoms according to Eqs. (3) and (6). Likewise, the dispersion curve of the periodic air rods for the TE mode is compared to the normalized SER of the transverse $(x y$-)polarized atoms, as shown in Fig. 4.

For the frequencies in the center of the photonic band gap, the SER in the $20 \times 20 \mathrm{PC}$ array can be reduced to less than $10^{-7}$ of that in free space for both the TM and TE cases. The (a)

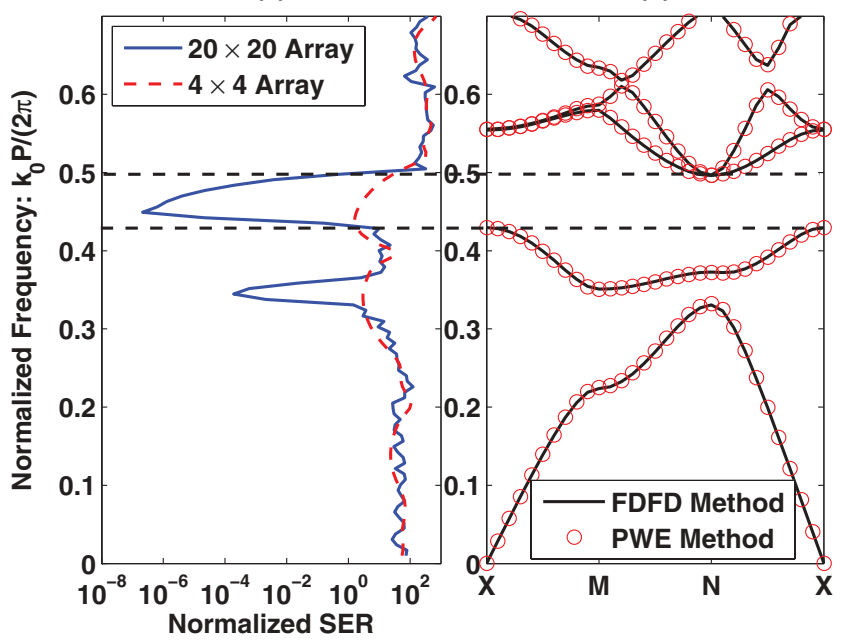

FIG. 4. (Color online) (a) The normalized SER of the atoms, which are located at the dielectric medium and surrounded by the $4 \times 4$ or $20 \times 20$ air rods. (b) The dispersion curve of the infinite PC structure with air rods for the TE mode. The SER is calculated by the summation of $\operatorname{Im}\left[G_{x x}\left(\mathbf{t}_{\mathbf{0}}, \mathbf{t}_{\mathbf{0}}\right)\right]$ and $\operatorname{Im}\left[G_{y y}\left(\mathbf{t}_{\mathbf{0}}, \mathbf{t}_{\mathbf{0}}\right)\right]$.

suppression of SE is caused by the Bragg scattering of the periodic PC array, where the LDOS of photons is extremely low and light cannot propagate. Ideally, no SE is allowed within the band gap of the infinite PC structure. Contrarily, the SE is significantly enhanced at the photonic band edge of the $20 \times 20$ PC array, where the group velocity of light is very slow and photons can couple to the radiation or leaky modes after a short mean free path. Thus, a high LDOS of photons can be achieved. Unlike the $20 \times 20$ PC array, the $4 \times 4$ PC array with few unit cells shows insignificant suppression or enhancement of SE because the coherent Bragg scattering becomes weaker and weaker. Hence, the plane-wave expansion algorithm [9], which implicitly imposes the periodic boundary condition or assumes infinite PC structure, cannot reproduce

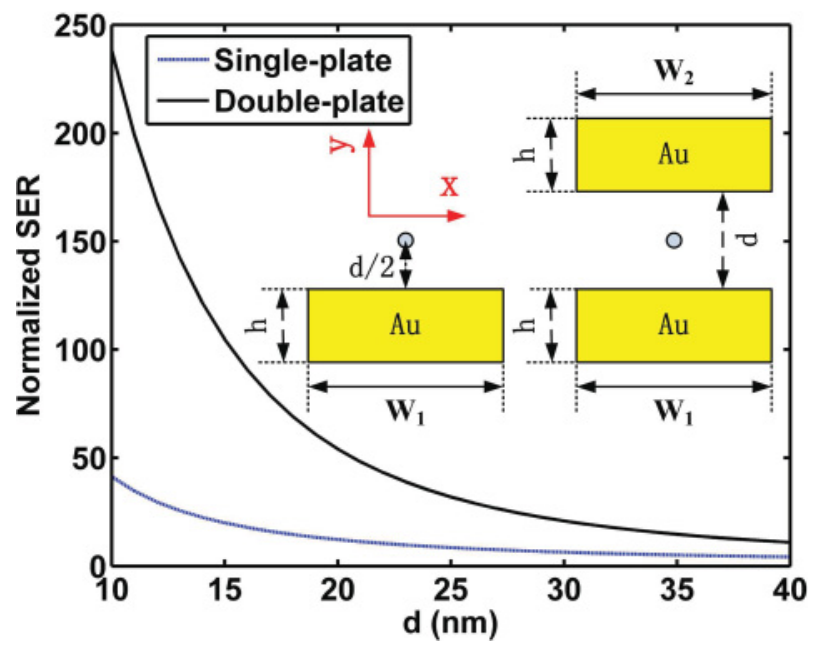

FIG. 5. (Color online) The normalized SER as a function of the distance between the atoms and the gold plates. The transition frequency of the atoms is set to be $510 \mathrm{~nm}$. 


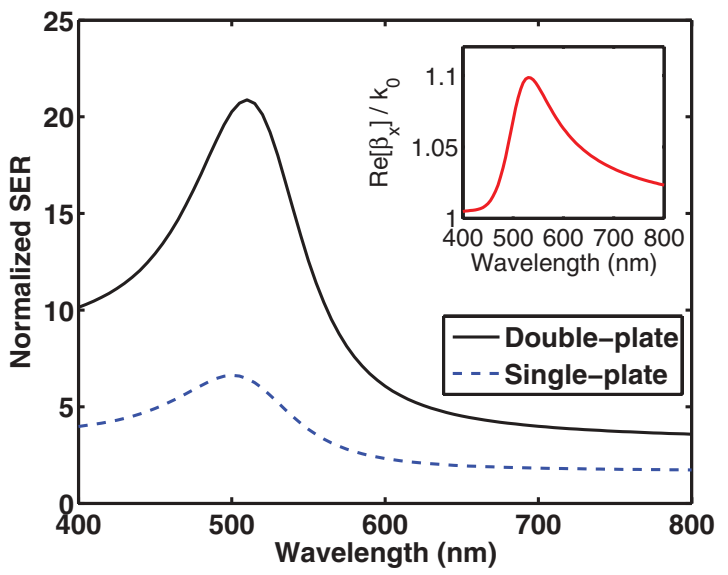

FIG. 6. (Color online) The normalized SER as a function of the transition frequency of the atoms. The distance $d$ is set to be $30 \mathrm{~nm}$.

the physics of SE in a finite PC structure with few unit cells.

(2) The SE of the atoms in the plasmonic metal plates is studied. The $z$-invariant gain media can be modeled as an ensemble of atoms or a line source. Figure 5 shows the schematic diagram; that is, the line source is located near a single Au plate or within a metal cavity by two Au plates. The widths of the bottom and top plates are $W_{1}=W_{2}=100 \mathrm{~nm}$, the thickness of each plate is $h=40 \mathrm{~nm}$, and the distance between two plates is $d$. The complex dielectric constant of $\mathrm{Au}$ is taken from [34]. When the location of the atoms moves close to the Au plate, the enhanced SE due to the surface plasmon resonance can be seen clearly in Fig. 5. Remarkably, the SE can be significantly boosted with the help of the metal cavity structure. The Purcell factor or the normalized SER can be almost 250 when the two metal plates are separated by $10 \mathrm{~nm}$, while it is less than 50 for the single metal plate. The extreme enhancement will be of great help for designing optoelectronic devices.

For the 2D semi-infinite metal-dielectric structure, only TE wave $\left(H_{z}, E_{x}, E_{y}\right.$ components) can excite the SPPs. The momentum (dispersion relation) of the SPP along the $x$-direction can be expressed as [35]

$$
\beta_{x}=k_{0} \sqrt{\frac{\epsilon_{m} \epsilon_{d}}{\epsilon_{m}+\epsilon_{d}}}
$$

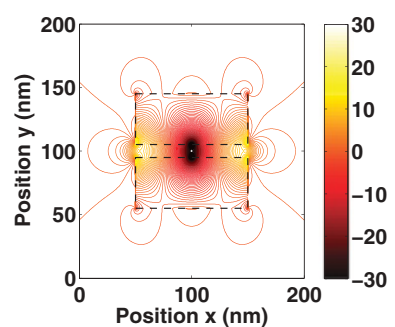

(a)

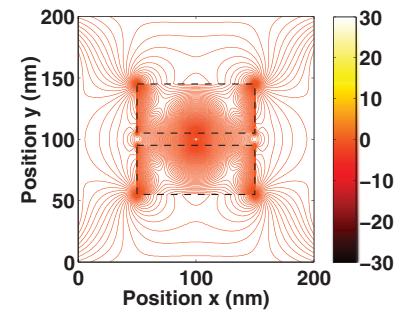

(b)

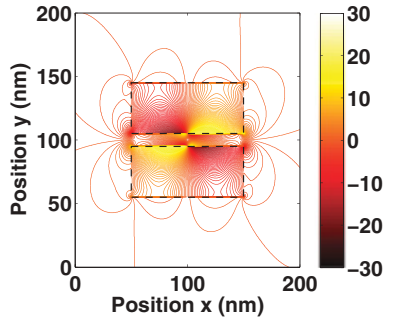

(c)

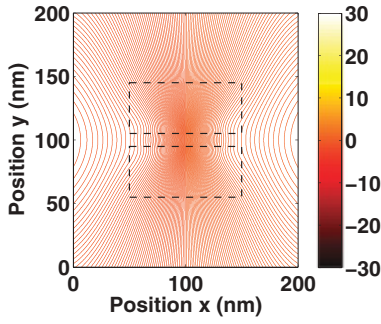

(d)

FIG. 7. (Color online) (a) $\operatorname{Im}\left[E_{y}\right]$ for $\hat{a}_{y}$ dipole source at $510 \mathrm{~nm}$; (b) $\operatorname{Im}\left[E_{y}\right]$ for $\hat{a}_{y}$ dipole source at $800 \mathrm{~nm}$; (c) $\operatorname{Im}\left[E_{x}\right]$ for $\hat{a}_{x} \operatorname{dipole}$ source at $510 \mathrm{~nm} ;(\mathrm{d}) \operatorname{Im}\left[E_{z}\right]$ for $\hat{a}_{z}$ monopole source at $510 \mathrm{~nm}$. 


\section{CONCLUSION}

Using the rigorous and efficient FDFD method, the SE in a 2D arbitrary inhomogeneous environment has been systematically studied. The electric DGF was obtained with the proper approximations of the monopole and dipole sources and with flexible treatments of inhomogeneous boundary conditions.

Under the investigation of both the PC and the plasmonic metal plate systems, we develop a universal concept that the SE strongly depends on the dispersion relation. In other words, the $\mathrm{SE}$ in an inhomogeneous environment can be engineered with dispersion management. Furthermore, the finite-structure or finite-size effects can modify the dispersion relation of electromagnetic system and should be exploited in the design of optoelectronic devices.

\section{ACKNOWLEDGMENTS}

The authors acknowledge the support of Grants No. 712108, No. 711508, and No. 711609 from the Research Grant Council of the Hong Kong Special Administrative Region, China. This project is also supported in part by a Hong Kong UGC Special Equipment Grant No. SEG HKU09.
[1] P. Lodahl, A. F. van Driel, I. S. Nikolaev, A. Irman, K. Overgaag, D. L. Vanmaekelbergh, and W. L. Vos, Nature (London) 430, 654 (2004).

[2] M. Fujita, S. Takahashi, Y. Tanaka, T. Asano, and S. Noda, Science 308, 1296 (2005).

[3] E. M. Purcell, Phys. Rev. Lett. 69, 681 (1946).

[4] A. A. Asatryan, K. Busch, R. C. McPhedran, L. C. Botten, C. M. de Sterke, and N. A. Nicorovici, Phys. Rev. E 63, 046612 (2001).

[5] D. P. Fussell, R. C. McPhedran, and C. Martijn de Sterke, Phys. Rev. E 70, 066608 (2004).

[6] A. A. Erchak, D. J. Ripin, S. Fan, P. Rakich, J. D. Joannopoulos, E. P. Ippen, G. S. Petrich, and L. A. Kolodziejski, Appl. Phys. Lett. 78, 563 (2001).

[7] M. Woldeyohannes and S. John, Phys. Rev. A 60, 5046 (1999).

[8] N. Vats and S. John, Phys. Rev. A 58, 4168 (1998).

[9] S. G. Johnson and J. D. Joannopoulos, Opt. Express 8, 173 (2001).

[10] A. Neogi, C. W. Lee, H. O. Everitt, T. Kuroda, A. Tackeuchi, and E. Yablonovitch, Phys. Rev. B 66, 153305 (2002).

[11] G. Sun, J. B. Khurgin, and R. A. Soref, Appl. Phys. Lett. 90, 111107 (2007).

[12] K. Okamoto, I. Niki, A. Shvartser, Y. Narukawa, T. Mukai, and A. Scherer, Nat. Mater. 3, 601 (2004).

[13] C. Walther, G. Scalari, M. I. Amanti, M. Beck, and J. Faist, Science 327, 1495 (2010).

[14] A. J. Ward and J. B. Pendry, Phys. Rev. B 58, 7252 (1998).

[15] V. Rao and S. Hughes, Opt. Lett. 33, 1587 (2008).

[16] G. Veronis and S. Fan, in Surface Plasmon Nanophotonics, edited by M. L. Brongersma and P. G. Kik, Chap. 12 (Springer, Dordrecht, The Netherlands, 2007), p. 170.

[17] A. Taflove and S. C. Hagness, Computational Electrodynamics: The Finite-Difference Time-Domain Method, 3rd ed. (Artech House, Boston, 2005).
[18] H. Y. D. Yang, IEEE Trans. Microwave Theory Tech. 44, 2688 (1996).

[19] W. C. Chew, Waves and Fields in Inhomogenous Media (Van Nostrand Reinhold, New York, 1990).

[20] L. Novotny and B. Hecht, Principles of Nano-Optics (Cambridge University Press, New York, 2006).

[21] K. Sakoda, Optical Properties of Photonic Crystals (Springer, Berlin, 2001).

[22] E. N. Economou, Green's Functions in Quantum Physics (Springer, Berlin, 2006).

[23] S. M. Barnett, B. Huttner, and R. Loudon, Phys. Rev. Lett. 68, 3698 (1992).

[24] Y. T. Chen, N. Gregersen, T. R. Nielsen, J. Mork, and P. Lodahl, Opt. Express 18, 12489 (2010).

[25] X. W. Chen, W. C. H. Choy, C. J. Liang, P. K. A. Wai, and S. He, Appl. Phys. Lett. 91, 221112 (2007).

[26] Y. Zhao, G. Wang, and X. H. Wang, J. Appl. Phys. 108, 063103 (2010).

[27] A. Rodriguez, M. Ibanescu, D. Iannuzzi, J. D. Joannopoulos, and S. G. Johnson, Phys. Rev. A 76, 032106 (2007).

[28] J. L. Xiong and W. C. Chew, Appl. Phys. Lett. 95, 154102 (2009).

[29] A. Brandt, Appl. Math. Comput. 19, 23 (1986).

[30] K. S. Yee, IEEE Trans. Antennas Propag. 14, 302 (1966).

[31] J. P. Berenger, J. Comput. Phys. 127, 363 (1996).

[32] W. C. Chew, J. M. Jin, and E. Michielssen, Microwave Opt. Technol. Lett. 15, 363 (1997).

[33] C. M. Rappaport, M. Kilmer, and E. Miller, Int. J. Numer. Modell. Electron. Networks Devices Fields 13, 471 (2000).

[34] A. D. Rakic, A. B. Djurisic, J. M. Elazar, and M. L. Majewski, Appl. Opt. 37, 5271 (1998).

[35] S. A. Maier, Plasmonics: Fundamentals and Applications (Springer, New York, 2007). 